\title{
Exploring Fusion Methods for Multimodal Emotion Recognition with Missing Data
}

\author{
Johannes Wagner, Florian Lingenfelser, Elisabeth André, and Jonghwa Kim, Senior Member, IEEE
}

\begin{abstract}
The study at hand aims at the development of a multimodal, ensemble-based system for emotion recognition. Special attention is given to a problem often neglected: missing data in one or more modalities. In offline evaluation the issue can be easily solved by excluding those parts of the corpus where one or more channels are corrupted or not suitable for evaluation. In real applications, however, we cannot neglect the challenge of missing data and have to find adequate ways to handle it. To address this, we do not expect examined data to be completely available at all time in our experiments. The presented system solves the problem at the multimodal fusion stage, so various ensemble techniques-covering established ones as well as rather novel emotion specific approaches-will be explained and enriched with strategies on how to compensate for temporarily unavailable modalities. We will compare and discuss advantages and drawbacks of fusion categories and extensive evaluation of mentioned techniques is carried out on the CALLAS Expressivity Corpus, featuring facial, vocal, and gestural modalities.
\end{abstract}

Index Terms_Ensemble based systems, decision-level fusion, multimodal emotion recognition, missing data.

\section{INTRODUCTION}

$\mathrm{E}$ MOTIONAL sensitivity in machines is believed to be a key element toward more human-like computer interaction. Due to the complex nature of human emotions, automatic emotion recognition still remains a challenging task after many years. One difficulty a machine has to face is the fact that humans rarely express their emotions exclusively, but use several channels such as speech and mimics. Studies that have focused on the fusion of multiple channels, however, often start from too optimistic assumptions, e.g., that all data from the different modalities is available at all times. As long as a system is only evaluated on offline data this assumption can be easily ensured by examining given samples beforehand and excluding parts where one or more channels are corrupted or are not suitable for evaluation. In real, application-oriented online systems, however, we cannot neglect the issue of missing data and have to find adequate ways to handle it so that robustness of recognition performance can be guaranteed.

Generally, we can identify various causes for missing data: A sensor device can fail so that an according signal is no longer available. Even if a sensor device is running properly, there is the possibility of desired information within a signal being no longer accessible, e.g., a tracked object disappears from the view of a camera. We can also think of a situation in which the desired information theoretically is at hand but is practically corrupted to some extent, e.g., a speech signal that is overlaid by noise. Finally, not only technical problems can

- The authors are with the Laboratory of Human-Centered Multimedia, University of Augsburg, Universitätsstr. 6a, 86159 Augsburg, Germany. E-mail: \{johannes.wagner, florian.lingenfelser, elisabeth.andre, jonghwa.kim)@informatik.uni-augsburg.de. be responsible for one or more modalities becoming useless. If a subject simply does not generate observable material, no meaningful data can be recorded, e.g., the gesture modality will not contribute relevant information while monitoring a momentarily motionless user. A system capable of handling missing data must therefore dynamically decide which channels are available and to what extent the present signals can be trusted. For the case where data are partly missing, a couple of treatments have been suggested in literature: Multiple imputation predicts missing values using existing values from previous samples [23]. In data marginalization, unreliable features are marginalized to reduce their effect during the decision process [11]. However, the consequences of imperfect data on the performance of fusion algorithms have not been systematically explored.

The realization of handling missing data within our aspired multimodal system is engineered within the fusion of available modalities. We explore various standard fusion schemes that are, among others, comprehensively described in [24], as well as being more sophisticated and emotion specific fusion techniques. All presented strategies are enriched with strategies on how to treat unavailable modalities.

For final evaluation, every online system can be pretrained with data gathered from multiple subjects and, afterward, classification tasks are carried out on a user not known during the training phase. An alternative to this user-independent approach is training the system with data recorded from the subject who is going to use the system afterward. This approach presumably adapts the system strongly to a single subject and therefore better classification results on this person can be expected, at the cost of universality. Both methods have their advantages and should be compared in terms of recognition accuracy.

The reported processing and classification methods, as well as all fusion-based approaches used in our experiments, have been developed with Smart Sensor Integration 
(SSI) [33], a framework for multimodal signal processing developed at our lab aiming to support the building of online recognition systems. SSI supports the design and evaluation of machine learning pipelines by offering tailored tools for signal processing, feature extraction, and pattern recognition, as well as tools to apply them offline (training phase) and online (real-time recognition).

\section{Related Work}

Recently, many studies in multimodal affect recognition have been done by exploiting a synergistic combination of different modalities. Most of the previous works focus on fusion of audiovisual information for automatic emotion recognition, e.g., combining speech with facial expression. De Silva et al. [28] and Chen et al. [10] proposed a rulebased decision level fusion method for a combined analysis of speech and facial expressions. Huang et al. [18] used boosting techniques to automatically determine adaptive weights for audio and visual features. In the work of Busso et al. [5], an emotion-specific comparison of feature-level and decision-level fusion has been reported by using an audiovisual database containing four emotions, sadness, anger, happiness, and neutral state, deliberately posed by an actress. They observed for their corpus that feature-level fusion was most suitable for differentiating anger and neutral state, while decision-level fusion performed better for happiness and sadness. They concluded that the best fusion method depends on the application. Interestingly, in addition to speech and facial expression, the thermal distribution of infrared images is also integrated into a multimodal recognition system [34] by considering the fact that infrared images are hardly affected by lighting conditions, which is one of the main problems in facial image analysis.

Humans use several modalities jointly in a complementary manner [10]. For decision-level fusion, however, multiple unimodal classifiers are trained for each modality separately and those decisions are fused by using specific weighting rules. This means that such a kind of fusion method is necessarily based on the assumption of conditional independence between modalities. To address this problem, a number of model level fusion methods have been proposed, originally in the research field of speaker identification, that are capable of exploiting cross-correlations between modalities. Song et al. [29], for example, proposed tripled HMM that models correlations between the upper face, lower face, and prosodic dynamic behaviors. By relaxing the general requirement of synchronized segmentation for audiovisual streams, Zeng et al. [35] proposed a multistream fused HMM which provides the possibility of optimal combination among multiple streams from audio and visual channels. For the estimation of correlation levels between the streams, they used the maximum entropy and the maximum mutual information criterion. Sebe et al. [27] suggest the use of dynamic Bayesian Networks to model the interdependencies between audio and video data and handle imperfect data by probabilistic inference. Finally, various types of multimodal correlation models are based on extended artificial neural networks, e.g., [14], [6].
Although there are many studies in psychology supporting combined face and body approaches as being the most informative for the analysis of human expressive behavior [1], there are surprisingly very few efforts reported on automatic emotion recognition by combining body gesture with other modalities such as facial expression and speech. For example, bimodal fusion methods at different levels for emotion recognition are presented by Balomenos et al. [2] and Gunes and Piccardi [15], using facial expression and body gesture. Kaliouby and Robinson [12] proposed a vision-based computational model to infer acted mental states from head movements and facial expressions. Castellano et al. [9] presented a multimodal approach for the recognition of eight emotions that integrates information from facial expressions, body gestures, and speech. They showed a recognition improvement of more than 10 percent compared to the most successful unimodal system and the superiority of feature-level fusion to decision-level fusion. All these approaches are based on visual analysis of expressive gestures and dealt with mapping different gesture shapes to relevant emotions. In our experiment, however, we use three-axis accelerometer instead of visual information in order to extract nonproportional movement properties, such as relative amplitude, speed, and movement fluidity, under the assumption that distinct emotions are closely associated with different qualities of body movement rather than gesture shapes.

All the studies reviewed above have shown that the performance of automatic emotion recognition systems can be improved by employing multimodal fusion. Some of them highlight the benefits of fusion mechanisms in situations with noisy features or missing values of features, for example, see [27]. Nevertheless, surprisingly few fusion approaches explicitly address the problem of nonavailable information. Most of them are based on the assumption that all data are available at all times. This precondition is not realistic in practical environments. In order to guarantee consistent classification, ways of handling missing sensory input have to be thought of.

\section{The CALLAS Expressivity Corpus}

For training and evaluation of classification systems the choice of an adequate corpus is substantial. Only significant empirical data enable meaningful statements about the performance of investigated recognition techniques. The CALLAS Expressivity Corpus [8] constitutes all desired demands for our aims. It was constructed within the European Integrated Project CALLAS and contains affective behavior, incorporating vocal utterances, facial expressions, and gesture expressivity in three primary emotion classes. The gesture stream is not available for all observations made, so applicable ways of handling missing data must be incorporated into experiments on this corpus.

\subsection{Data Generation}

As the present corpus was originally designed for examination of cultural differences between emotion expressions of persons from different European countries, it was initially made with subjects from Greece, Italy, and Germany. This work is based solely on data collected from 


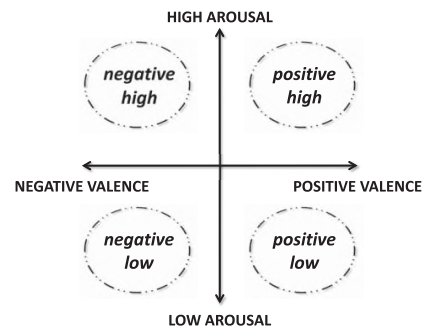

Fig. 1. Arousal valence-based emotion model.

German participants, as we do not aim at dealing with cultural differences in detail. The German subcorpus contains 21 people (10 female and 11 male) and almost $5 \mathrm{~h}$ of recorded interaction, ${ }^{1}$ which is sufficient for our investigations.

During the experiment, participants were asked to perform expressive sentences through voice, face, and body language. During a session, 120 emotion-inducing sentences were successively displayed to the participants. The sentences, which were inspired by the Velten mood induction technique [31], can be divided according to their semantic content in three broad categories, namely, positive, negative, and neutral. After he or she had read a sentence silently, the projection was blanked out and the sentence got expressed in their own words and with whatever gesture or voice they felt to be fitting. It should be noted that recorded people had no acting background and it was left to their discretion to what extent they expressed the emotions. This, of course, leads to a broader diversity among observed expressions than it would under a more restrictive setup. However, it comes closer to what a system must expect under a realistic setting. When, in some situations, subjects were not using gestures to accompany their speech at all, this just reflects what happens in real life, and hence renders a situation an emotion recognition system must deal with. For our experiments, no samples were removed from the data set.

While users were performing the sentences, their actions were captured with two cameras, one focused on the proband's face and the other on the whole body. In the following progress, we only analyze videos captured from the face and refer to it as the facial modality. Voice was captured from a microphone hanging above the user's head. Gestures were tracked in three different modes: either free handed by the body-camera, a Humanware data-glove on one hand, or two Nintendo Wii remote controls, one in each of the user's hands. For the purpose of the study at hand, we only use data gathered by the Wii controller for movement and acceleration tracking.

\subsection{Modeling Emotions}

In order to deal with multimodal emotion recognition, a concept of discrete emotion modeling has to be chosen. The term emotion itself is a very abstract concept, describing a vast amount of human feelings. These feelings are too numerous to use them directly for recognition tasks, so they have to be integrated into quantifiable categories of emotions. A discrete emotion model is necessary to define target emotions so that the recognition system is able to

1. Since user sessions were continuously captured, this includes intermediate parts where users were reading sentences or changing devices.

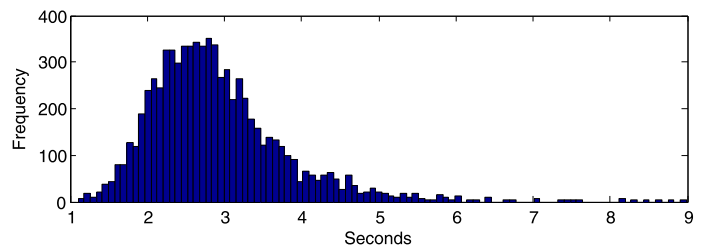

Fig. 2. Histogram of segment lengths.

understand the problem to be solved. Moreover, the emotion model supports a convergent labelling process. Such procedures narrow the amount of identifiable feelings and group the wide field of possible individual emotions into a small amount of discrete emotion-classes. One possibility is to have all recorded emotional experiences labeled by external specialists and subsumed under predefined expressions like love, hate, sadness, surprise, etc. However, this approach could be restricting, as many blended feelings and emotions cannot adequately be described by the chosen categories. Selection of some particular expressions cannot be expected to cover a broad range of emotional states and could suffer from randomness. Another way of categorizing emotions is to attach the stimuli experienced to continuous scales. Lang [21] proposes arousal and valence as measurements (see Fig. 1). These scales describe multiple aspects of an emotion, the combination of stimuli's alignments on these scales defines single emotions. More precisely, the valence scale describes the pleasantness of a given emotion. A positive valence value indicates an enjoyable emotion such as joy or pleasure. Negative values are associated with unpleasant emotions like sadness and fear. This designation is complemented by the arousal scale, which measures the agitation level of an emotion. Combination of the two scales forms four emotion-quadrants, representing a four-class classification problem to be dealt with by a emotion recognition system.

\subsection{Segmentation and Annotation}

As explained earlier, users were asked to utter expressive sentences and accompany them with whatever gesture or voice they felt to be fitting. As a natural consequence of this experimental design the voice channel became the dominant modality, which usually triggered on and offset of a performance. Only in a very few cases were mimics or gestures observed before or after an utterance. Consequently, we decided to segment the streams according to the speech signal and used the beginning and ending of each utterance of a sentence as borders. In this way, we ended up with 2,513 segments, ${ }^{2}$ each containing according snapshots of available audio, video, and acceleration streams. On average, these segments have a length of 2.9 a with a standard deviation of 0.9 a. Fig. 2 shows a histogram of the distribution.

When annotating a multimodal corpus, we must decide which of the available modalities should serve as the source throughout the labeling process. In the case of the corpus at hand, judgments could be based either exclusively on the audio or video channel or by presenting both modalities simultaneously. We did decide in favor of the audio channel as it is-in consequence of the chosen segmentation

2. Seven segments were skipped either because subjects refused to perform or due to technical problems. 
procedure-the only channel that is always available throughout the whole corpus. Labels assigned to the speech chunks are then consequently applied to the other modalities. In doing so, we actually neglect a problem that often comes across in social communication, namely, the problem of blended and masked emotions, which leads to ambiguous expressions across modalities. For instance, if we consider a situation where we are forced to talk with calm voice while at the same time we use mimics to express our anger about something. However, since such a situation is unlikely to occur in the present corpus, we assume that emotions are more or less homogeneously expressed across the three modalities.

Though the used mood inducing sentences are more categorized along the valence axis (positive, neutral, and negative), it becomes obvious that an arousal categorization is also needed. Especially when looking at negative sentences, there are samples tending to a depressed and sad mood (negative-low), while others are expressed in an aroused and angry way (negative-high). Nearly all neutral and part of the positive observations share a calm and optimistic subtone (positive-low) in contrast to fewer positive examples bearing clear hints of joy and laughter (positivehigh). Based on these impressions, we refrained from including neutral moods as their own class and label calm and nonnegative emotions as positive-low. For annotating recorded samples, three experts were asked to independently label the observations made in terms of high or low arousal as well as positive or negative valence. The term experts is used to denote that the raters have some sort of knowledge about emotions and their recognition that goes beyond everyday experience. During the annotation phase, audio segments were replayed in chronological order to each expert independently. Raters could loop an utterance as often as needed and even jump forth and back in order to repeat older segments and reassign labels.

Final combination of differing annotations is done via majority decision; as three assessments are given to each orientation of valence or arousal, respectively, decisions are definite. For instance, if the first rater assigns label low and positive, the second low and negative, and the third high and negative, the segment is finally labeled as low and negative. Applying the described voting, we end up with 1,145 samples labeled as positive-low, 527 as positive-high, 608 as negative-low, and 233 as negative-high.

To report inter-rater reliability, we calculate the kappa value according to Fleiss [13]. Fleiss's Kappa value is a common way to measure the agreement over multiple raters. It is expressed as a number between 0 and 1 , where 1 indicates a perfect agreement. Applied to the decision of our raters, we get for valence a kappa value of 0.84 , which indicates an almost perfect agreement. For arousal, on the other side, we measure a notably lower value of 0.38 , which implies a fair agreement. This drift obviously follows from the fact that expressed sentences were selected to be either negative, neutral, or positive. Thus, it should be easier for the raters to agree on the valence of an utterance than its level of arousal. The kappa value for all four classes amounts to 0.52 , which expresses a moderate agreement. The obvious disagreement of the raters regarding the labels that were assigned to the samples makes it already clear that we must not reckon 100 percent accuracy in classification.

\section{Methodology}

In this section, we introduce feature extraction methods for available modalities and describe the classification scheme and feature-selection strategies we applied to the CALLAS corpus. As we mainly focus on building online recognition systems, we solely rely on methods that can be computed within a feasible amount of time. In particular, feature extraction and classification should be applicable in (near) real-time and must not require manual tuning at runtime.

\subsection{Classification Model}

Naive Bayes (NB) is a simple but efficient classification scheme. It is based on the Bayes Theorem, which states

$$
P\left(E_{i} \mid f_{1}, \ldots, f_{n}\right)=\frac{P\left(E_{i}\right) \prod_{j=1}^{n} P\left(f_{j} \mid E_{i}\right)}{P\left(f_{1}, \ldots, f_{n}\right)} .
$$

In other words, this means that the probability of the emotion $E_{i}$ given an observed feature vector $\left(f_{1}, \ldots, f_{n}\right)$ of dimension $n$ depends on the a priori probability $P\left(E_{i}\right)$ of the emotion, multiplied by the product of the probability of each feature $f_{i}$ given the emotion, divided by the a priori probability of the feature vector. As a classification result, the emotion $E_{i}$ from a set of $N$ emotions $E_{1}, \ldots, E_{N}$ that maximizes the equation is chosen. This is simplified (and hence the name Naive Bayes) as the Bayes Theorem assumes the features to be independent of each other. Parameters for the probability distributions $P\left(E_{i}\right)$ and $P\left(f_{j} \mid E_{i}\right)$ are gained from the annotated training material.

Some of the fusion algorithms tested in this paper combine results from no less than 12 internal classification models. To run experiments in a reasonable amount of time, it was more important for us to rely on a fast classification scheme than one that gives highest classification rates. Hence, we have chosen Naive Bayes as it is extremely fast in training and testing, even for high-dimensional feature vectors and large training databases. Using a more sophisticated, but at the same time much slower classifier, such as Support Vector Machine (SVM), would certainly improve recognition accuracies. However, since the main focus of this study is the comparison of fusion strategies, the underlying classification model is of less importance.

\subsection{Feature Extraction}

The extraction of descriptive features brings the raw signals into the compact form required by the classifier. Often features are computed after a preprocessing phase during which additional properties of the signals are carved out and unwanted aspects are suppressed. Depending on whether the features are extracted on a small running window of fixed size or for longer chunks of variable length, we denote them as short or long-term features. While, in offline analysis, the whole signal is available from the beginning and processing can fall back on global statistics, such as global mean and standard-deviation, or perform zero-phase filtering by processing the input data in both the forward and reverse directions, such treatments are not possible in online 
TABLE 1

Overview of Preprocessing Steps and Feature Extraction Methods Applied in Our Experiments

\begin{tabular}{|c|c|c|c|c|c|}
\hline modality & channels & $\begin{array}{l}\text { pre- } \\
\text { processing }\end{array}$ & short-term feature & long-term feature & total \\
\hline voice & $\begin{array}{l}\text { mono } \\
\text { audio, } \\
16 \mathrm{kHz}\end{array}$ & $\begin{array}{l}\text { pre- } \\
\text { emphasis } \\
\text { filter }\end{array}$ & $\begin{array}{l}\text { pitch, energy, MFCCs, spec- } \\
\text { tral, voice quality }\end{array}$ & $\begin{array}{l}\text { mean, median, maximum, minimum, vari- } \\
\text { ance, median, lower/upper quartile, abso- } \\
\text { lute/quartile range }\end{array}$ & 1316 \\
\hline face & $\begin{array}{l}\text { RGB video, } \\
720 \times 576 \\
25 \mathrm{fps}\end{array}$ & $\begin{array}{l}\text { conversion } \\
\text { to gray } \\
\text { image }\end{array}$ & $\begin{array}{l}\text { bounding box of face, } \\
\text { position of eyes, mouth } \\
\text { and nose, opening of } \\
\text { mouth, facial expression } \\
\text { happy/angry/sad/surprised }\end{array}$ & $\begin{array}{l}\text { mean, energy, standard deviation, min- } \\
\text { imum, maximum, range, position mini- } \\
\text { mum/maximum, number crossings/peaks, } \\
\text { length }\end{array}$ & 264 \\
\hline gesture & $\begin{array}{l}3 \text { axes ac- } \\
\text { celeration, } \\
50 \mathrm{fps}\end{array}$ & $\begin{array}{l}\text { remove } \\
\text { trend }\end{array}$ & $\begin{array}{l}\text { acceleration and 1st deriva- } \\
\text { tive, velocity, position }\end{array}$ & $\begin{array}{l}\text { power, fluidity, volume, mean, minimum, } \\
\text { maximum, position minimum/maximum, } \\
\text { length }\end{array}$ & 75 \\
\hline
\end{tabular}

processing. In our experiments, signals are processed in small blocks with a fixed window size. ${ }^{3}$ This implies that only the information of the current and previously seen blocks are available to our algorithms. Table 1 offers a summary of the applied processing methods. A detailed description is given in the following paragraphs.

\subsubsection{Speech Features}

The CALLAS corpus includes mono audio recordings from a single USB microphone (Samson C01U) placed near the subject's head. The audio stream was captured at a sample rate of $16 \mathrm{kHz}$ and quantized by 16 bit PCM. Recording quality and noise level were similar among all sessions.

The list of proposed features suitable for emotion recognition from speech is long. A cooperation of different sites under the name CEICES (Combining Efforts for Improving Automatic Classification of Emotional user States) has carried out experiments based on a pool of more than 4,000 features, including acoustic and linguistic feature types [3]. Results for the individual groups, as well as a combined set, have led to the following assumptions: Among acoustic features, duration and energy seem to be most relevant, while voice quality showed less impact. Yet, no single group outperformed the pool of all acoustic features.

In our experiments, we restricted the set of features to those that can be extracted in real time and in a fully automatic manner. For example, no features have been included that require information on the spoken words or grammatical context, as such information is difficult to get without manual annotation. Hence, we only compute acoustic features related to the paralinguistic message of speech, i.e., we analyze "how" something is said. In our previous studies [32], MFCC and spectral features turned out to be good candidates. In addition, we also compute features from pitch, energy, duration, voicing, and voice quality, and use feature selection to reduce the full set of 1,316 features to the most relevant ones. In a previous study, the feature set was evaluated on the Berlin Database of Emotional Speech [4] that is commonly used in offline research (7 emotion classes, 10 professional actors) and

3. At each processing step, a small portion of the signal equal to the window size is processed. Afterward, the window is moved by a certain number of samples, which is defined by the frame shift. achieved an average recognition accuracy of 80 percent. On the FAU Aibo Emotion Corpus [30], as part of the INTERSPEECH Emotion Challenge 2009 [25], we were able to slightly exceed the baseline given by the organizers for a five class problem (anger, emphatic, neutral, positive, and rest) [32]. Both corpora have been intensively studied in the past by many researchers working on emotion recognition from speech and serve as a kind of benchmark in this area.

\subsubsection{Facial Features}

As mentioned earlier, we use only video recordings of the subject's face. The according camera was placed in a distance of about $2 \mathrm{~m}$ and captured frames include a close-up of shoulder and head. The resolution of the video is $720 \times 576$ pixels at $25 \mathrm{fps}$. Videos are stored in uncompressed 24-bit RGB format. Video processing is provided by SHORE, a library for facial emotion detection developed by Fraunhofer IIS ${ }^{4}$ [20]. In the first place, SHORE offers a robust tracking of in-plane rotated faces up to 60 degrees. For each face that is found, SHORE reports the bounding box of the face, as well as position of the left/right eye and the nose tip. These features measure head movement. In addition, the left and right corners of the mouth and its degree of opening is reported. Most importantly, SHORE also calculates scores for four facial expressions, namely, happy, angry, sad, and surprised. These scores are also extracted for each frame and used in addition to the geometric features. In total, for each segment, a series of 24 short-term features is derived by joining features extracted for each frame in the clip. Finally, we extract 11 long-term measurements, leading to an overall feature set with 264 entries.

\subsubsection{Gestural Features}

The acceleration sensors included in the Wii remote control include a three-axis linear accelerometer, which measures the force exerted by a set of small proof masses inside of it with respect to its enclosure. To categorize gestural style, we can rely on expressivity parameters, e.g., how fast a gesture is done, how much space one uses to perform a gesture, etc. In a previous study, we tested the feasibility of this approach on a training set containing 1,260 samples by 7 subjects [26]. Using a 10-fold cross-validation of a Nearest

4. http://www.iis.fraunhofer.de/en/bf/bv/ks/gpe/demo/. 
Neighbor classifier, we obtained recognition results of at least 94 percent for power, speed, and spatial extent.

The features we extract from the acceleration signal go back to a set of expressivity parameters originally defined by Hartman et al. for expressive gesture synthesis for embodied Conversational Agents [17]. Caridakis et al. have applied similar features to measure gesture expressivity in hand tracking from video images [7]. They propose a set of six expressivity parameters, namely, overall activation, spatial extent, temporal, fluidity, power/energy, and repetitivity. Overall activation, e.g., is considered as the overall quantity of movement, while fluidity differentiates smooth/graceful from sudden/jerky gestures.

Since we do not have direct access to the $2 \mathrm{D}$ position in space, but instead measure the second derivation, i.e., acceleration, we apply a couple of changes to their algorithm. First, we eliminate the influence of gravity by removing the linear trend from each of the three acceleration axes. Next, we build the first derivative and use cumulative trapezoidal numerical integration to deduce velocity and position. This is done for each axis separately. Finally, we calculate the power from each signal, as well as fluidity from position. We also extract another seven statistical features from the acceleration signal and add the length of the gesture. Overall, we obtain 75 features from the three acceleration axes.

\subsection{Feature Selection}

Especially on small corpora, a large number of features can lead to a problem known as the "curse of dimensionality." This term was introduced by Richard Bellman in 1961 as mathematical problem, and in machine learning it describes the exponentially rising need for numbers of samples for a sufficient description of a high-dimensional feature space. In short, this means that the more features that are given to a classification model, the more samples that are needed to train it. In most cases, however, not all of the features add useful information to the classification problem, while some may carry redundant information. This fact is related to the challenge of finding that subset of features which tweaks the best recognition performance.

To select a set of the most relevant features in a feasible amount of time, we apply a combination of two selection approaches. First, we select the best 150 features according to correlation-based feature subset selection (CFS, [16]). CFS aims at finding a subset of features where the correlation of each feature with the class is maximized while the correlation of the features among each other is low. This strategy is especially beneficial for the Naive Bayes classifier, which performs badly when features are highly correlated since it assumes features to be independent for simplification reasons.

Afterward, sequential forward selection (SFS) is applied on this subset and stopped after 100 iterations (i.e., after 100 features have been selected). SFS is a simple, but popular selection method. Like other wrapper approaches, it uses a classifier to measure the performance gain of different feature subsets. SFS starts from an empty subset and adds, at each step, the feature that brings the highest performance gain. In order to avoid overfitting, crossvalidation should be used to evaluate the feature sets. Finally, the subset which by then gives the best performance is selected.

\subsection{Recognizing Missing Data}

As described earlier, the CALLAS corpus is well-suited for exploring the problem of missing data. The gesture modality is partially missing or no movements of hands were executed during a sample. The facial modality is missing at points in time when SHORE lost track of a recorded person and therefore no meaningful facial features could be extracted. As the recordings of samples start and end concurrently with a mood inducing sentence, the vocal modality is the only source that is always available. Handling of missing data is modeled within the multimodal fusion process. Recognition of missing data has to be executed beforehand. Therefore, we keep track of the time when SHORE loses the bounding box around an observed face-this happening marks recorded data as missing until the face is recognized again. We furthermore introduce a threshold for minimum energy within a signal recorded from the Wii controller and, whenever energy falls below this mark, we assume that no gesture was performed at all during the recorded phrase.

\section{Multisensor Data Fusion}

The examined CALLAS data set consists of up to three modalities-video, speech, and gesture data. As a special challenge, channels are not always accessible, so dedicated ways of fusing all available data channels have to be thought of. In order to discuss preconditions and advantages of decision-level fusion in a multisensor environment, we begin with a description of differences between the possible levels on which fusion can be executed and follow up with precise reviews on possible decision level fusion methods and inherited strategies meant to deal with missing data.

\subsection{Feature-Level Fusion}

Feature-level fusion is a common and straightforward way to fuse all recorded observation channels. All desired features are merged into a single high-dimensional feature set. One single classifier is then trained for the task of classification. As the fused data contain a larger amount of information than single modalities, an increase in classification accuracy can theoretically be expected. In practice, these classifiers yield reliable classification results. But this very accessible approach to data fusion comes along with a couple of major problems: The first drawback is the eventually occurring curse of dimensionality on small data sets (see Section 4.3). If the available data are not ample, the classification results become nonmeaningful. As the second, it has to be mentioned that a growing feature vector may stress computational resources for training and evaluation of the classification model. In some examinations, these obstacles may be not of interest due to a fair availability of time and resources; other ones may refuse the feature level approach solely because of these reasons and consider decision level approaches to data fusion instead.

A very crucial shortcoming of the feature fusion approach can be observed in particular on the CALLAS data set. The single classifier trained on the whole feature set is, by default, not capable of handling the problem of missing data. Furthermore, the feature level fusion approach does not give the opportunity to employ any strategies like elaborating the 
fusion strategy for affective tasks. The behavior of featurelevel fusion is strictly determined by the selected feature-set and underlying classification model.

\subsection{Decision-Level Fusion}

Contrary to feature-level fusion and its reliance on a single classifier that deals with a high-dimensional feature vector, decision-level fusion focuses on the usage of small classifiers and their combination. Instead, the available feature set is divided into subgroups and the partitions are used to form several small classification models (of course, these classifiers can also be generated by subsampling training data or the usage of different classification models). The assembly of these classifiers is called an ensemble. Outcomes of these slim classifier models are taken into account for the final decision making process. The term decision-level fusion sums up a variety of methods designed in order to merge the decisions of ensemble members into one single ensemble decision.

\subsubsection{Ensemble-Based System}

Classification models used in creating the underlying ensemble for all discussed decision-making algorithms stem from multimodal emotion observations using facial, vocal, and gesture recordings. Our implemented system forms an ensemble by providing features from each listed channel with a classification model. They must neither provide perfect performance on some given problem nor do their outputs need to resemble each other. It is preferable that the chosen classifiers make mistakes, at best on different instances. A base idea of ensemble-based systems is to reduce the total error rate of classification by strategically combining the members of the ensemble and their errors. Therefore, the single classifiers need to be diverse from one another.

\subsubsection{Benefits of Decision-Level Fusion}

Ensemble-based systems and corresponding decision-level fusion offer some significant advantages over the use of a single classifier. A few important ones should be listed here.

Training efficiency. In many applications, a vast amount of data is gathered and computational efficiency can greatly suffer from training and evaluation of a single classifier with huge data sets. Partitioning of data, training of independent classifiers with different subsets, and combination for a final decision often proves to be more practical, time-saving, and yields at least competitive results in most cases. ${ }^{5}$ Given the contrary case where too little data are available, various resampling techniques can be used to form overlapping subsamples of the data set. Each of the resulting subsets can be applied for training of classifiers which then are capable of decision making via combination.

Divide and conquer. Another classification problem can arise if the underlying data set and the corresponding feature distribution is too complex for a sole classifier to learn. Classification accuracy seriously suffers if the needed decision boundary cannot be found by the used classification

5. Several smaller classifiers may not save training time when using a classification model of linear time complexity (e.g., Naive Bayes- $O(n)$ ) as the training will consume as much time as for an overarching classifier. But as time complexity rises (e.g., Support Vector Machines classification scheme (SVM)-O $\left(n^{2}\right)$ or worse), this behavior changes in favor of small classifiers, using only a section of the original, high-dimensional feature vector or training data. model. This undesirable phenomenon can be counteracted by an appropriate set of classifiers. Using a divide-andconquer approach, the feature space is divided into several (perhaps overlapping) distributions that are easier to learn. Each of these partitions is then handled by one classifier. Adapted combination of the gained classifiers and their simplified decision boundaries adequately simulates the original, complex boundary.

Field performance. The training and testing of classification models typically takes place on data gained from some kind of laboratory environment. Statements about generalized classification performance experienced in field testing-whenever previously unknown samples appearare difficult to estimate. The risk of performing below average in the field is much higher for a single classifier than for an assemblage of classifier models. Some bychance poorly trained classifiers within a set are much less of a menace than a single classifier performing poorly.

Concerned with systems for multisensor data fusion and real-time applications, these can be implemented in such a way that they resist the breakdown of one or more attached sensors. If the classifiers involved in decision making each represent the observations of an associated sensory device, the absence of a single contribution to the final decision is unlikely to result in a drastic quality fall-off for overall classification accuracy, especially if the sensory malfunction is recognized and the corresponding classifier's (most likely counter-productive) contribution is rated accordingly.

\subsubsection{Decision Level Fusion Techniques}

Having established an ensemble-based system, the diverse decisions of its members have to be merged into a single ensemble decision. For this purpose, we can choose from various established fusion strategies. Among the strategies, we find all forms of algebraic combinations of the classifiers continuous outputs, ranking methods, as well as varying voting schemes and other ways of class label combination. One feature of some discussed methods is the appliance of weights to the ensemble members. Based on prior knowledge-like, for example, gained by evaluation of training performance-single classifiers can be associated with a certain weight. This way, their importance within the ensemble is reflected. Note the immense importance of not taking any knowledge of data to be classified into account for the calculation of mentioned weights. Otherwise, unrealistic prior knowledge is hypothesized and regarded experimental results can no longer be rated as significant. We apply described fusion schemes to modalities given by the CALLAS corpus. As our system handles temporarily ${ }^{6}$ missing modalities in the fusion step, all described fusion methods are enriched with strategies on how to handle missing data streams.

For the explanation of reviewed algorithms the following annotations are used: The decision of ensemble member $t$ for class $n$ is denoted as $d_{t, n} \in\{0,1\}$, with $t=1 . . T$ and $n=$ $1 . . N$ and $d_{t, n}=1$ if class $\omega_{n}$ is chosen, $d_{t, n}=0$, otherwise. Respectively, the support given to each class $n$ (i.e., the

6. Temporarily means that we adjust the fusion scheme per input sample (not, as in most fusion approaches, for the whole corpus), i.e., for each modality and each sample we decide whether to include the information to the fusion process or not. At the moment, this decision is exclusive. If we had some way of estimating the degree of corruption within the sample's modality, we could simply assign proper weights instead. 
calculated probability for the observed sample to belong to single classes) by classifier $t$ is described as $s_{t, n} \in[1 . .0]$.

Weighted Majority Voting. Majority Voting simply sums up decisions of $T$ classifiers. The ensemble decision for an observed sample $x$ is chosen to be the class $\omega_{n}$, which received the most votes (decisions) $v_{n}$. A definite decision is only guaranteed if an odd number of ensemble members handle a two-class problem. In Weighted Majority Voting each vote is associated with the precalculated weight of the ensemble member. The ensemble decision for an observed sample $x$ is chosen to be the class $\omega_{n}$ which received the most weighted votes $v_{n}$. Ties are not likely to happen this way, which makes the weighted variant more suited for practical application:

$$
v_{n}(x)=\sum_{t=1}^{T} w_{t} d_{t, n}(x) .
$$

Handling missing data. Ensemble member $t$ containing training data from modalities not featured in an observed sample is not included in the poll.

Weighted Average. In contrast to Weighted Majority Voting, the Weighted Average strategy applies weights not to class labels, but to continuous outputs of ensemble members. By summing up the weighted support given to each class $\omega_{n}$, total weighted support $\mu_{n}$ for class $n$ is calculated as

$$
\mu_{n}(x)=\sum_{t=1}^{T} w_{t} s_{t, n}(x) .
$$

The ensemble decision for an observed sample $x$ is chosen to be the class $\omega_{n}$ for which support $\mu_{n}(x)$ is largest.

Handling missing data. Ensemble member $t$, containing training data from modalities not featured in an observed sample, is weighted with a value of zero.

Maximum Rule, Minimum Rule, Median Rule. These strategies choose the maximum, minimum, or median support generated by $T$ ensemble members. The ensemble decision for an observed sample $x$ is chosen to be the class $\omega_{n}$ for which support $\mu_{n}(x)$ is largest.

Handling missing data. Ensemble member $t$, containing training data from modalities not featured in an observed sample, gives support $s_{t}$ of value zero to each of the $n$ classes.

Sum Rule, Mean Rule. The Sum Rule simply sums up the support given to each class $\omega_{n}$ in order to generate total support $\mu_{n}$ for each class. The ensemble decision for an observed sample $x$ is chosen to be the class $\omega_{n}$ for which support $\mu_{n}(x)$ is largest.

By averaging the support $\left(\frac{1}{T}\right.$ serves as normalization factor) given to each class $\omega_{n}$, the Mean Rule calculates total support $\mu_{n}$ for class $n$ as

$$
\mu_{n}(x)=\frac{1}{T} \sum_{t=1}^{T} s_{t, n}(x) .
$$

Handling missing data. Ensemble member $t$, containing training data from modalities not featured in an observed sample, gives support $s_{t}$ of value zero to each of the $n$ classes.

Product Rule. By multiplying the support given to each class $\omega_{n}$, total support $\mu_{n}$ for class $n$ is calculated as

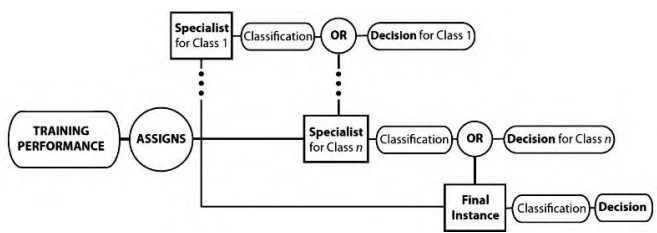

Fig. 3. Cascading specialists scheme.

$$
\mu_{n}(x)=\frac{1}{T} \prod_{t=1}^{T} s_{t, n}(x) .
$$

Note that this fusion strategy reacts very sensitively to pessimistic ensemble members as a support of value zero virtually nullifies the chance of a class becoming the final decision.

Handling missing data. Ensemble member $t$, containing training data from modalities not featured in an observed sample, gives support $s_{t}$ of value 1 to each of the $n$ classes.

Cascading Specialists. The Cascading Specialists method [22] does not focus on merging outputs from all ensemble members, but on selecting experts for each class and bringing them in a reasonable order (see Fig. 3). Based on evaluation of training data, experts for every class of the classification problem are chosen. Next, classes are rank ordered, from worst classified class across the ensemble's members to the best one. Given these preparations, classification works as follows: First, class in the sequence is chosen and the corresponding specialist is asked to classify the sample. If the output matches the currently observed class, this classification is chosen as ensemble decision. If not, the sample is passed on to the next weaker class and corresponding expert while repeating the strategy. Whenever the case occurs that none of the experts classifies its connected class, the classifier with the best overall performance on the training data is selected as the final instance and is asked to label the sample. This strategy aims at a flattening effect among class accuracies that will, at best, improve overall classification performance.

Handling missing data. The concept of choosing experts for certain classes has to be broadened so that an expert unable to handle the given sample (because of missing data) can be adequately replaced. Instead of selecting one single ensemble member for expert and final classification tasks, ordered lists containing all classifiers_ranked by their qualification for the given task-replace sole classifiers. If, in the classification step, missing data are detected and the most qualified ensemble member is trained with data of that type, we move down in the prepared list to find the next best classifier that is able to handle the observed sample.

Arousal-valence combination. The generic approach to classification in a multiclass environment is to train classifiers and corresponding ensembles to categorize among the available classes, but the structure, the chosen emotion model-consisting of two scales for valence and arousal-more emotion-adapted techniques for finding an ensemble decision can be applied [19]: Two ensembles are trained to recognize the observed emotion's axial alignment. Resulting outputs are logically combined for final decision, as decisions on valence and arousal orientations explicitly describe one of specified classes. Because of the 


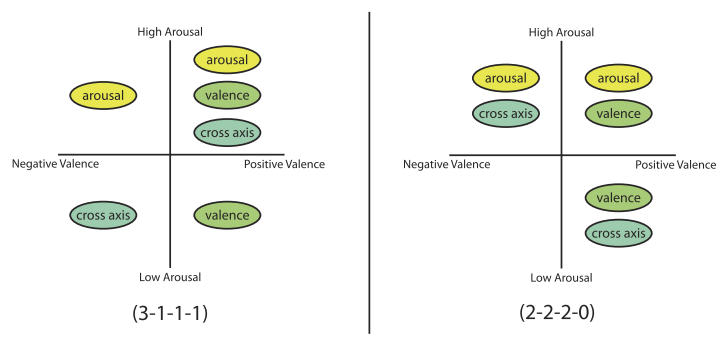

Fig. 4. Possible vote distributions after Step 1 after arousal-valence combination.

mapping to orientation in the emotion model, this strategy cannot be generalized for common classification problems.

Handling missing data. In our implementation, Weighted Majority Voting is used to generate the ensembles' decisions on axial alignments. Therefore, the handling of missing data described for the respective fusion scheme is adopted.

Arousal-valence cross-axis combination. The concept of a cross-axis again is exclusive to the chosen emotion model and is meant to provide supplementary information to the arousal and valence ensembles. This axis cannot be directly deduced from emotion theory, but, from a mathematical point of view, it is a reasonable partition of the given $2 \mathrm{D}$ space. Just like the arousal and valence axes, the cross-axis divides the emotion model into two separate parts, each containing two emotion-quadrants. These parts contain the respective, complementary quadrants and therefore split the model in a diagonal way. According to arousal and valence, a proper ensemble for cross-axis is constructed. A stepwise algorithm is used for combination of the sources of information ${ }^{7}$ :

In Step 1, each ensemble distributes its votes among the two quadrants that fit the recognized alignments in the emotion model (see Fig. 4). This step results in one of two possible outcomes:

If the ensembles agree on one emotion-quadrant, it receives three votes and can already be chosen as the final decision; otherwise, a voting tie occurs. No final decision can be chosen; instead the draw has to be dissolved and the algorithm moves on to Step 2. A direct classification ensemble designates exactly one vote to the class it predicts. Two situations can arise through this supplemental vote: If the ensemble chooses an emotion-quadrant that already holds two votes, the tie is resolved and the corresponding emotion is determined to be the final decision. Otherwise, the ensemble chooses the emotion-quadrant that has not yet received any votes, the tie is not resolved, and Step 3 to be executed: The emotion-class that was originally determined by arousal and valence ensembles is chosen as the final decision. In practice, this case rarely occurs, but it is definitely needed to guarantee that no sample passes the decision process unclassified.

Handling missing data. Again, Weighted Majority Voting and respective handling of missing data is used in all ensemble decisions.

7. An additional ensemble for direct classification-as established for generic approaches-is needed for Step 2 of the combination strategy.

\section{EXPERIMENTS}

The experiments presented are done on the described CALLAS Expressivity corpus. Missing data are included in the facial and gestural modality-the vocal modality is always accessible-as samples represent one spoken sentence. In detail, audio signals are constantly present in all 2,513 samples, facial features can successfully be extracted throughout 2,251 observations (90 percent), and significant gestures are available for 569 samples ( 24 percent). In consequence of the experimental design, the samples are more or less equally distributed among the 21 subjects; however, if we calculate the relative portion of samples per emotion, we find a high variety between the users. In general, female users recorded in this experiment seem to perform more expressively than their male counterparts.

\subsection{Evaluation Method}

Choosing an adequate evaluation method is crucial for meaningful experiments. Among others, possibilities involve random drawing of samples for testing, percentaged subset drawing, $k$-fold splits, or the leave-one-out strategy. As this work focuses strongly on the practical adaptability of presented methods and a good field performance under life-like or even disadvantageous circumstances, the chosen evaluation method should reflect this intention. We agreed on a very realistic, user-independent approach for evaluation of our experiments (Leave-One-Speaker-Out). As the employed corpus contains 10 female and 11 male participants, we consecutively draw samples belonging to one single subject out of the set. The remaining samples are used for training of classification models, which then are tested against the isolated samples. Another important decision concerns the way recognition rates are presented. In consequence of the dominant presence of positive-low and a general imbalance in class-distribution, we base our studies on the classwise recognition rate (sometimes referred to as unweighted average recall), which is the mean of the recognition rates observed for each class.

\subsection{Discussion}

Results shown in Table 2 are split into three parts: First, single channel performance is shown for each modality. Whenever missing data are found, the respective sample is not included for evaluation, so these results stem from different quantities of samples. Vocal modality clearly outperforms facial and gestural cues and establishes the most balanced accuracies among observed classes, though positive emotions are recognized better than negative ones. The facial modality recognizes the positive-high emotion very well—presumably because it is well suited for detection of smiles and movements of the face associated with laughter-but lacks on other classes. Gestures are most often correctly classified during positive-low phases, the most calmly expressed class of observed emotional states with nearly no movement at all. Negative-low emotions were often expressed with despairing gestures that could be partially separated from gestures with high arousal. These expressive movements were obviously often misinterpreted among each other, leading to very low accuracies on classes with highly aroused emotions. 
TABLE 2

Results Achieved for Single Modalities in Comparison with Decision-Level Fusion and Emotion-Adapted Fusion

\begin{tabular}{rccccc}
\hline & \multicolumn{5}{c}{ Recognition Results } \\
\cline { 2 - 5 } & p-low & p-high & n-low & n-high & avg \\
\hline \multicolumn{5}{c}{ Single Modalities } \\
Voice & 0.61 & 0.50 & 0.49 & 0.43 & $\mathbf{0 . 5 1}$ \\
Face & 0.45 & 0.72 & 0.31 & 0.43 & $\mathbf{0 . 4 8}$ \\
Gesture & 0.57 & 0.30 & 0.44 & 0.36 & $\mathbf{0 . 4 2}$ \\
\hline Generic Decision Level Fusion \\
Cascading & 0.49 & 0.63 & 0.47 & 0.40 & $\mathbf{0 . 5 0}$ \\
MaxRule & 0.55 & 0.65 & 0.36 & 0.46 & $\mathbf{0 . 5 0}$ \\
MeanRule & 0.57 & 0.66 & 0.41 & 0.37 & $\mathbf{0 . 5 0}$ \\
MedianRule & 0.58 & 0.67 & 0.41 & 0.38 & $\mathbf{0 . 5 1}$ \\
MinRule & 0.59 & 0.58 & 0.48 & 0.33 & $\mathbf{0 . 4 9}$ \\
ProdRule & 0.58 & 0.66 & 0.42 & 0.38 & $\mathbf{0 . 5 1}$ \\
SumRule & 0.57 & 0.66 & 0.41 & 0.37 & $\mathbf{0 . 5 0}$ \\
WeightAvg & 0.53 & 0.63 & 0.39 & 0.41 & $\mathbf{0 . 4 9}$ \\
WeightMajVote & 0.61 & 0.52 & 0.49 & 0.42 & $\mathbf{0 . 5 1}$ \\
\hline Emotion & Adapted Decision Level Fusion \\
\hline ArousalValence & 0.56 & 0.46 & 0.55 & 0.51 & $\mathbf{0 . 5 2}$ \\
CrossAxis & 0.64 & 0.55 & 0.56 & 0.44 & $\mathbf{0 . 5 5}$ \\
\hline \multicolumn{7}{c}{}
\end{tabular}

The second part of Table 2 shows the results of generic fusion approaches that can be applied to any given classification problem. Theoretically, all decision level fusion strategies aim at exploiting mentioned differences in single channels in order to enhance combined performance. Practically, performances of facial and gestural modalities are too inferior to the audio channel to result in greater gains in overall recognition rates. If one compares the vocal modality to the fusion schemes, most approaches perform better on the positive-high class. This behavior can be explained by the very good result of the facial modality in this area and the resulting influence on the ensemble. Unfortunately, bad results for the remaining classes do effect overall performance in a contrary way and these gains are lost again in other categories. This can be well observed when looking at the Product and Sum Rule - the "standard" fusion schemes for merging classifier outputs-as recognition results stabilize around vocal performance, with a better trend on the second class. The same estimations hold for other merging strategies like Mean or Weighted Average Rule. The behavior of approaches that choose exactly one support value among ensemble members for each class (Max, Median, and Min Rule) is harder to predict, but all of them resemble the just-mentioned characteristics and their overall performances range from worst to best results within the generic fusion category. Weighted Majority Voting's inherent weighting method causes a strong reliance on the dominant modality, resulting in almost the same accuracies across all classes as the audio channel. The Cascading Specialists strategy generates acceptable results on negative classes-that are all in all more weakly categorized throughout the ensemble- but loses too much accuracy on the positivelow class in order to improve average accuracy. However, no strategy generates drastically worse results than the best modality - actually, they perform well compared to the remaining modalities.

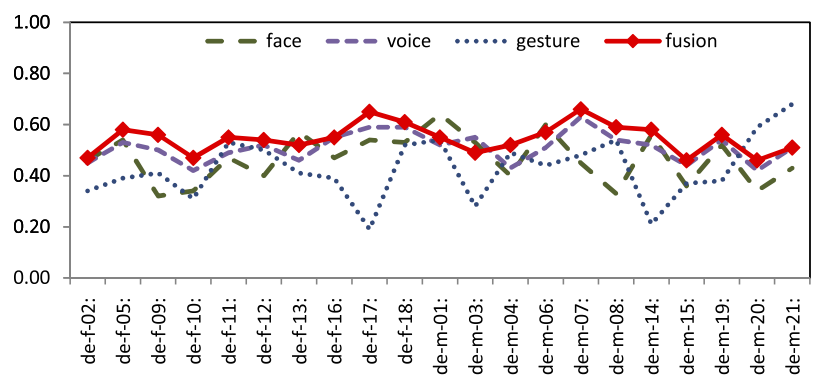

Fig. 5. Recognition performance per user of single-channel classification and the fusion approach (user-independent).

So, in order to perceptibly enhance recognition rates compared to single channel classification on the dominant modality, we have to exploit deeper knowledge about the classification problem at hand with emotion-adapted fusion strategies that employ more than a single generic ensemble. The combination of arousal and valence ensembles shows different characteristics than the generic approaches: The dominance of the positive-high class is gone and negative classes are well recognized. Overall, these changes result in slightly superior accuracy than the best single channel. For further improvements, we incorporate more available information from the 2D emotion model into combination strategies, leading to the additional cross-axis ensemble. This fusion scheme exceeds the best modality on every observed class and therefore enhances average accuracy remarkably, however, at the expense of a rising ensemble count.

Of course, the observed results cannot be claimed to be universal as they are highly corpus-dependent. Unfortunately, by the time this paper was written, there was-at least to our best knowledge-no publicly available emotion corpus, which could suit our needs in terms of modalities, naturalness and presence of missing data. Such a corpus could serve as a benchmark to compare presented methods with results achieved by other institutions and to prove generality. However, observations are in line with an earlier study based on a smaller emotional corpus, where emotionspecific fusion approaches clearly outperformed generic ones [19].

\subsection{Single-Channel Classification}

To learn more about the contribution of the single modalities to the overall performance, Fig. 5 shows classwise recognition rates for each channel and user (dashed lines). User names are encoded in the form de- $[f, m]-i d$, where $f$ denotes a female and $m$ a male user. The line charts reveal high variability in all three modalities. The most extreme difference occurs in the gesture channel ranging from 19 percent (de-f-17) to 68 percent (de-m-21). For audio and video, the differences are slightly more stable, but still within a range of 21 percent and 31 percent, respectively. The results give no hints for a general preference of female and male users for a certain channel. In fact, it appears to be rather randomly distributed as to which are the strong channels of the single users. There are also no indications of a correlation between the channels, i.e., a low/high performance in one channel does not necessarily indicate a low/high performance in the other channels. 


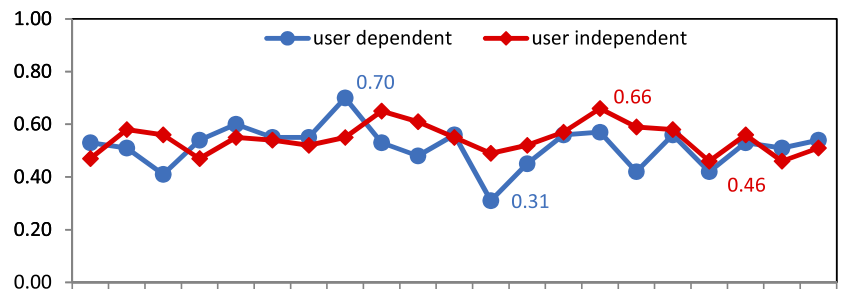

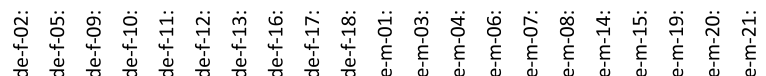

Fig. 6. Recognition rates for single-user-dependent classification (blue line) and for user-independent classification (red line).

Fig. 5 additionally reveals that the fusion approach (red line) improves the results achieved by the best single channel for 15 out of 21 subjects. For the remaining subjects (de-f-13, de-m-01, de-m-03, de-m-06, de-m-20, de-m-21), decision-level fusion evens results, while it still outperforms the other two channels. Nevertheless, as fusion strategies consider all modalities, there is always a chance that a failure in one channel affects good performance of other ones. This is the tradeoff for balancing weak modalities and corrupted data. After all, ensemble-based strategies always guarantee results that are-if not superior-at least in line with the dominating modality. This characteristic is especially desirable, when the most trustworthy modality for a user is not known in advance.

\subsection{Single-User Dependent Classification}

The reasons for relying on user independent evaluation were already explained in Section 6.1. It may be still be worthwhile to draw a comparison to single-user dependent classification. We surely would expect better classification performance from a system trained and tested with samples of the same user. In order to prove this assumption, we select all samples of each user and investigate recognition rates for each user independently by five-fold cross-evaluation. Results are visualized in Fig. 6.

Some remarkable facts have to be mentioned: First, one can observe a high variability in overall performance, ranging from 31 percent to 70 percent in single-user dependent classification, whereas results in user-independent classification are a bit more stable ranging from 46 percent to 66 percent. Obviously, the results of singleuser dependent classification are not necessarily superior to the user-independent approach chosen for our evaluations. In fact, for a noticeable amount of users (e.g., de-f- 09 and de-m-03) overall classification rates prove to be significantly lower. ${ }^{8}$ Finally, we observe a common trend in userindependent and single-user-dependent evaluation, i.e., high performance in the single-user-dependent classification implies high performance in the user-independent case and the other way round.

The observations made suggest that the success of singleuser-dependent as well as user-independent emotion recognition always strongly correlates with the expressivity of investigated subjects. Unfortunately, we have to reckon with a high variety in expressivity among users. Further,

8. Note that the amount of training samples available for userindependent classification is about 20 times higher compared to singleuser dependent evaluation. results suggest that a system trained on a considerable large database of prerecorded users can yield similar results as a system trained on a limited set of personalized samples collected from the user.

\section{Conclusion}

In this work, we report results of decision level fusion experiments carried out on the CALLAS Expressivity corpus, performing emotion recognition from three different modalities, namely, voice, face, and gesture. Classification accuracies of single modalities range from 42 percent to 51 percent while appropriately recognizing and dealing with missing data in observed channels. By means of ensemble techniques, results were raised up to 55 percent, including various generic fusion schemes as well as emotion adapted approaches like the combination of arousal, valence, and cross-axis. We see that generic approaches adapt to the most dominant modality in the ensemble while adopting some characteristics of other ensemble members-like the good performance on positive-high from the video channel. Exploiting the structure of the underlying emotion model leads to more elaborate fusion strategies-including the usage of more than one ensemble- that are tailored for affective emotion recognition. These fusion schemes do not share the aforementioned characteristics and are able to outperform single modalities and generic approaches by a significant rate, though bearing a higher complexity due to the generation of specialized ensembles.

Comparing recognition results of the single subjects we found a high variability within a range of more than 30 percent. This correlates with a high variety in expressivity among users. This variability also appears when observing results for the single channels. In fact, we could not identify a general trend that would suggest one channel to be more important than another. Though the vocal modality looks more stable overall, the facial channel is more suited to certain users. Even the gesture channel, as the weakest of the three channels, outperformed the others for some users. The uncertainty about the channel a user picks to express his or her emotion, however, is a strong argument for the benefit of fusion in our experiment as it reduces the risk of trusting a weak modality.

As another interesting outcome, we found that the results obtained for user-independent classification also yielded similar and sometimes even better results than single-user dependent classification. Hence, an emotion recognition system trained with data of a large number of subjects must not necessarily achieve worse results than a personalized system trained with a small number of training samples from the user. Ideally, the system would start with a pretrained model, which is, over time, adapted to the user. However, we also observed that a low performance in the single-user dependent classification implies low performance in the user independent case, i.e., the success of an emotion recognition system always depends to a large part on the expressivity of the user.

For future work, we also aim at improving the segmentation techniques applied to the CALLAS corpus. So far, the recorded modalities have been segmented in a very straightforward way. Based on the user's speech, the beginning and ending of a recorded sample coincide with 
the boundaries of the spoken stimuli sentence. Facial and gestural signals are simply observed and segmented over the according time span. This strategy suffers from two major problems: The hopefully expressed emotion could occur within a much shorter period somewhere within the spoken sentence and therefore information before and afterward is not of great meaning for recognition. Furthermore, significant hints from different modalities are not guaranteed to emerge at exactly the same time interval. Classification accuracy could be expected to improve if modalities were segmented individually and the succession and corresponding delays between occurrences of emotional hints in different signals could be investigated more closely. However, this approach gives room for a whole new set of hypotheses and experiments.

\section{ACKNOWLEDGMENTS}

The work described in this paper is funded by the European Union under research grant CALLAS (IST-34800) and CEEDS (FP7-ICT-2009-5).

\section{REFERENCES}

[1] N. Ambady and R. Rosenthal, "Thin Slices of Expressive Behavior as Predictors of Interpersonal Consequences: A Meta-Analysis," Psychological Bull. vol. 111, no. 2, pp. 256-274, 1992.

[2] T. Balomenos, A. Raouzaiou, S. Ioannou, S. Drosopoulos, A. Karpouzis, and S. Kollias, "Emotion Analysis in Man-Machine Interaction Systems," Proc. Workshop Machine Learning for Multimodal Interaction, 2005.

[3] A. Batliner, S. Steidl, B. Schuller, D. Seppi, K. Laskowski, T. Vogt, L. Devillers, L. Vidrascu, N. Amir, L. Kessous, and V. Aharonson, "Combining Efforts for Improving Automatic Classification of Emotional User States," Language Technologies, T. Erjavec, and J. Gros, eds., pp. 240-245, 2006.

[4] F. Burkhardt, A. Paeschke, M. Rolfes, W. Sendlmeier, and B. Weiss, "A Database of German Emotional Speech," Proc. Interspeech Conf., pp. 1517-1520, 2005.

[5] C. Busso, Z. Deng, S. Yildirim, M. Bulut, C.M. Lee, A. Kazemzadeh, S. Lee, U. Neumann, and S. Narayanan, "Analysis of Emotion Recognition Using Facial Expressions, Speech and Multimodal Information," Proc. Int'l Conf. Multimodal Interfaces, pp. 205-211, 2004.

[6] G. Caridakis, L. Malatesta, L. Kessous, N. Amir, A. Paouzaiou, and K. Karpouzis, "Modeling Naturalistic Affective States via Facial and Vocal Expression Recognition," Proc. Int'l Conf. Multimodal Interfaces, pp. 146-154, 2006.

[7] G. Caridakis, A. Raouzaiou, K. Karpouzis, and S. Kollias, "Synthesizing Gesture Expressivity Based on Real Sequences," Proc. Workshop Multimodal Corpora, 2006.

[8] G. Caridakis, J. Wagner, A. Raouzaiou, Z. Curto, E. André, and K. Karpouzis, "A Multimodal Corpus for Gesture Expressivity Analysis Multimodal Corpora," Proc. Workshop Multimodal Corpora, 2010.

[9] G. Castellano, L. Kessous, and G. Caridakis, "Multimodal Emotion Recognition from Expressive Faces, Body Gestures and Speech," Proc Doctoral Consortium Second Int'l Conf. Affective Computing and Intelligent Interaction, 2007.

[10] L.S. Chen, T.S. Huang, T. Miyasato, and R. Nakatsum, "Multimodal Human Emotion/Expression Recognition," Proc. Int'l Conf. Automatic Face and Gesture Recognition FG, p. 366, 1998.

[11] C. Demiroglu, D.V. Anderson, and M.A. Clements, "A Missing Data-Based Feature Fusion Strategy for Noise-Robust Automatic Speech Recognition Using Noisy Sensors," Proc. Int'l Symp. Circuits and Systems, pp. 965-968, 2007.

[12] R. El Kaliouby and P. Robinson, "Generalization of a Vision-Based Computational Model of Mind-Reading," Proc. Int'l Conf. Affective Computing and Intelligent Interfaces pp 582-589, 2005.

[13] J.L. Fleiss, B. Levin, and M.C. Paik, Statistical Methods for Rates and Proportions, third ed. John Wiley \& Sons, 2003.
[14] F. Fragopanagos and J.G. Taylor, "Emotion Recognition in Human-Computer Interaction," Neural Networks, vol. 18, no. 4, pp. 389-405, 2005.

[15] H. Gunes, M. Piccardi, and T. Jan, "Face and Body Gesture Recognition for a Vision-Based Multimodal Analyzer," Proc. PanSydney Area Workshop Visual Information Processing, pp. 19-28, 2004.

[16] M.A. Hall, "Correlation-Based Feature Subset Selection for Machine Learning," master's thesis, Univ. of Waikato, Hamilton, New Zealand, Apr. 1998.

[17] B. Hartmann, M. Mancini, and C. Pelachaud, "Implementing Expressive Gesture Synthesis for Embodied Conversational Agents," Proc. Second Conf. Int'l Soc. Gesture Studies, 2005.

[18] L. Huang, L. Xin, L. Zhao, and J. Tao, "Combining Audio and Video by Dominance in Bimodal Emotion Recognition," Proc. Affective Computing and Intelligent Interaction, pp. 729-730, 2007.

[19] J. Kim and F. Lingenfelser, "Ensemble Approaches to Parametric Decision Fusion for Bimodal Emotion Recognition," Proc. Int'l Conf. Bio-Inspired Systems and Signal Processing, pp. 460-463, 2010.

[20] C. Küblbeck and A. Ernst, "Face Detection and Tracking in Video Sequences Using the Modified Census Transformation," J. Image and Vision Computing, vol. 24, no. 6, pp. 564-572, 2006.

[21] P.J. Lang, M.M. Bradley, and B.N. Cuthbert, "Motivated Attention: Affect, Activation, and Action," Attention and Orienting: Sensory and Motivational Processes, P.J. Lang, R.F. Simons, and M.T. Balaban, eds., pp. 97-135, Erlbaum, 1997.

[22] F. Lingenfelser, J. Wagner, T. Vogt, J. Kim, and E. André, "Age and Gender Classification from Speech Using Decision Level Fusion and Ensemble Based Techniques," Proc. Interspeech Conf., 2010.

[23] M. Pantic and L. Rothkrantz, "Toward an Affect-Sensitive Multimodal Human-Computer Interaction," Proc. IEEE, vol. 91, no. 9, pp. 1370-1390, Sept. 2003.

[24] R. Polikar, "Ensemble Based Systems in Decision Making," IEEE Circuits and Systems Magazine, vol. 6, no. 3, pp. 21-45, 2006.

[25] B. Schuller, S. Steidl, and A. Batliner, "The INTERSPEECH 2009 Emotion Challenge," Proc. Interspeech Conf., pp. 312-315, 2009.

[26] M. Rehm, N. Bee, and E. Andé, "Wave Like an Egyptian Accelerometer Based Gesture Recognition for Culture Specific Interactions," Proc. Conf. HCI 2008 Culture, Creativity, Interaction, 2008.

[27] N. Sebe, I. Cohen, T. Gevers, and T.S. Huang, "Multimodal Approaches for Emotion Recognition: A Survey," Proc. SPIE Conf., pp. 56-67, 2004.

[28] L.C. De Silva and P.C. Ng, "Bimodal Emotion Recognition," Proc. Int'l Conf. Automatic Face and Gesture Recognition, pp. 332-335, 2000.

[29] M. Song, J. Bu, C. Chen, and N. Li, “Audio-Visual Based Emotion Recognition: A New Approach," Proc. IEEE Conf. Computer Vision and Pattern Recognition, pp. 1020-1025, 2004.

[30] S. Steidl, "Automatic Classification of Emotion-Related User States in Spontaneous Children's Speech," PhD thesis, Logos Verlag, Germany, 2009.

[31] E. Velten, "A Laboratory Task for Induction of Mood States," Behavior Research and Therapy, vol. 35, pp. 72-82, 1998.

[32] T. Vogt and E. André, "Exploring the Benefits of Discretization of Acoustic Features for Speech Emotion Recognition," Proc. Interspeech Conf., pp. 328-331, 2009.

[33] J. Wagner, E. André, and F. Jung, "Smart Sensor Integration: A Framework for Multimodal Emotion Recognition in Real-Time," Proc. Affective Computing and Intelligent Interaction, 2009.

[34] Y. Yoshitomi, S. Kim, T. Kawano, and T. Kitazoe, "Effect of Sensor Fusion for Recognition of Emotional States Using Voice, Face Image, and Thermal Image of Face," Proc. Int'l Workshop RobotHuman Interaction, 2000.

[35] Z. Zeng, J. Tu, B. Pianfetti, M. Liu, T. Zhang, Z. Zhang, T.S. Huang, and S. Levinson, "Audio-Visual Emotion Recognition through Multi-Stream Fused HMM for HCI Applications," Proc. IEEE Conf. Computer Vision and Pattern Recognition, pp. 967-972, 2005. 


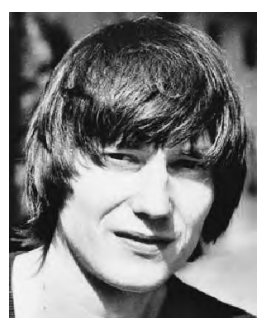

Johannes Wagner received the MSc degree in informatics and multimedia from the University of Augsburg, Germany, in 2007. Afterward, he joined the chair for Human-Centered Multimedia of the same university. Among other projects, he has been working on multimodal signal processing in the framework of CALLAS and is currently developing a general framework for the integration of multiple sensors into multimedia applications called Social Signal Interpretation (SSI).

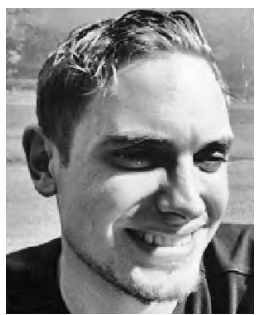

Florian Lingenfelser received the MSc degree in informatics and multimedia from the University of Augsburg, Germany, in 2009. In 2010, he joined the chair for Human-Centered Multimedia of the same university as a PhD student. He is currently contributing to multimodal data fusion within the CEEDS project and developing the Social Signal Interpretation framework.

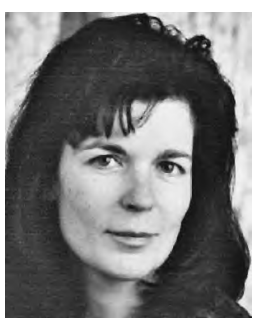

Elisabeth André is a full professor of computer science at Augsburg University and chair of the Laboratory for Human-Centered Multimedia, Augsburg University, Augsburg, Germany. Earlier, she worked as a principal researcher at DFKI GmbH, where she led various academic and industrial projects in the area of intelligent user interfaces. In summer 2007, she was nominated a Fellow of the Alcatel-Lucent Foundation for Communications Research. In 2010, she was elected a member of the prestigious German Academy of Sciences Leopoldina and the Academy of Europe. Her research interests include affective computing, intelligent multimedia interfaces, and embodied agents.

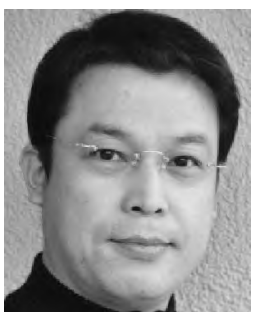

Jonghwa Kim received the BS and MS degrees in electronic engineering from Kyungwon University, Korea, in 1992 and 1994, respectively. He completed the diploma in sound engineering and received the $\mathrm{PhD}$ degree in communication engineering from the Technical University of Berlin, Germany, in 1999 and 2003, respectively. In 2002, he joined the Institute of Computer Science at the University of Augsburg, Germany, and finished the Habilitation (Venia Legendi) for computer science in 2010. He is a senior member of the IEEE. 